\title{
Manual Lymph Drainage as a Complex Decongestive Therapy for Upper Limb Lymphedema After Breast Cancer Operation
}

\author{
Ma Yu-hua ${ }^{1}$, Guo Xiao-xia ${ }^{2,}$, , Zhang Li-tao ${ }^{1}$, Lv Rong-zhao ${ }^{1}$, Li Shi-ting ${ }^{1}$, Tang Wan ${ }^{1}$ \\ ${ }^{1}$ Department of Breast Surgery, The First Affiliated Hospital of Jinan University, Guangzhou, China \\ ${ }^{2}$ The First Affiliated Hospital of Jinan University, Guangzhou, China
}

Email address:

guoxiaoxia2019@sina.com (Guo Xiao-xia)

${ }^{*}$ Corresponding author

To cite this article:

Ma Yu-hua, Guo Xiao-xia, Zhang Li-tao, Lv Rong-zhao, Li Shi-ting, Tang Wan. Manual Lymph Drainage as a Complex Decongestive Therapy for Upper Limb Lymphedema After Breast Cancer Operation. Journal of Family Medicine and Health Care.

Vol. 5, No. 4, 2019, pp. 45-49. doi: 10.11648/j.jfmhc.20190504.12

Received: August 15, 2019; Accepted: September 10, 2019; Published: October 9, 2019

\begin{abstract}
Objective We aim to explore the clinical effect of manual lymph drainage as a complex decongestive therapy on improving upper limb lymphedema after breast cancer operation. Methods Manual lymph drainage was performed on 28 patients with lymphedema after receiving breast cancer operation. Circumference measurement was done at transverse palmar crease, transverse carpal crease, $10 \mathrm{~cm}$ below transverse cubital crease, $10 \mathrm{~cm}$ above transverse cubital crease and $20 \mathrm{~cm}$ above transverse cubital crease at the first day before treatment and 1 day, 1 week, 2 weeks and 3 weeks after treatment. The movement of shoulder joints, pain and numbness of patients were recorded. Results After 3 weeks of manual lymph drainage, the circumference at transverse palmar crease, transverse carpal crease, $10 \mathrm{~cm}$ below transverse cubital crease, $10 \mathrm{~cm}$ above transverse cubital crease and $20 \mathrm{~cm}$ above transverse cubital crease was significantly lower than that before treatment. The detumescence of the middle segment of forearm $(10 \mathrm{~cm}$ below transverse cubital crease) was better than that of the middle segment of upper arm $(10 \mathrm{~cm}$ above transverse cubital crease $)$ and the superior segment of upper arm $(20 \mathrm{~cm}$ above transverse cubital crease) with a significant difference $(\mathrm{P}<0.05)$. After the treatment of manual lymph drainage, the life quality of patients improved greatly and there was a significant difference $(\mathrm{P}<0.05)$. Conclusion Manual lymph drainage as a complex decongestive therapy for lymphedema after breast cancer operation is safe, effective and thus well worth clinical application.
\end{abstract}

Keywords: Lymphedema, Manual Lymph Drainage, Complex Decongestive Therapy, Breast Cancer

\section{Introduction}

Breast cancer is the most common cancerous diseases among women $[1,2]$. Currently, the major treatment for invasive breast cancer are operations, most of which is modified radical operation. When modified radical operation is performed, axillary lymph node dissection and postoperative radiotherapy of local lesions will undermine lymphatic net, causing lymphedema. It is reported that the incidence of upper limb lymphedema after radical mastectomy is $13.6 \%-20.2 \%$ [3]. If no intervention is given to lymphedema, pain in the affected upper limb, edema, numbness, chill and high fever will appear and finally lead to abnormal skin appearance, infection and axillary web syndrome, lowering the quality of patients' life [4, 5]. Clinically, postoperative functional exercise is adopted to reduce the incidence of lymphedema but it has limited effect. At present, complex decongestive therapy (CDT) has become one of the standard treatments for lymphedema and has been effectively applied to postoperative lymphedema [6]. CDT includes personalized skin care, professional manual lymph drainage, low-elastic bandaging and functional exercise of the affected limb, etc. Since December 2014, our department has performed complex decongestive therapy (CDT) for lymphedema. Patients complicated with breast lymphedema after undergoing breast-conserving therapy were given manual lymph drainage. The decongestive intervention effectively promotes return of the lymph that is stagnated in 
the breast tissues and reduces axillary web syndrome. The study is reported as follows.

\section{Data and Methods}

\subsection{General Data}

The Breast Surgery of the First Affiliated Hospital of Jinan University admitted 28 female patients with upper limb lymphedema after breast cancer operation from December 2014 to December 2018. The inclusion criteria are: all patients are above 18 years old (including 18); the lymphedema appears on the same side of the breast cancer; all participants are fully aware of their diagnosis, volunteer for the study and give their informed consent, and they can accept and understand the health coaching; complete data of the patients can be obtained; all patients received CDT treatment. Exclusion criteria include: terminal breast cancer, recurrence, distant metastasis and metastatic breast cancer; those with other major organ diseases or severe general infection, severe anemia, etc.; patients with abnormal cognitive behavior and having used antidepressant in the recent past; edema induced by thrombus or tumor embolus; patients likely to have local infection, ulcer and bleeding.

\subsection{Interventions}

Professional nurses responsible for lymphedema treatment in the General Surgery Department of the First Affiliated Hospital of Jinan University evaluated, diagnosed and performed manual lymph drainage on all the patients once per day and1.5h per time for 15 times in a 21-day period (except Saturday and Sunday).

\subsubsection{Skin Care}

The nurses checked whether there was a wound, ulcer, infection or other diseases in patients' skin, and exhorted the patients to use natural neutral body wash for skin cleansing. Besides, patients should avoid rubbing the skin vigorously to protect the skin from breaking and ensure the skin folds clean.

\subsubsection{Opening Up Lymphatic Pathways}

Patients totally relaxed and assumed a sitting or horizontal position. Professional nurses used pulps of index finger, middle finger and ring finger to gently move the skin covering superficial lymph nodes as "stationary circles" to open up supraclavicular, infraclavicular, cervical, preauricular, retroauricular, parasternal, axillary, inguinal lymph nodes and thoracic lymph nodes on the cancerous side. The massage was done from the healthy side to cancerous side and the pressure was about $25 \mathrm{mmHg}$ [6].

\subsubsection{Relief of Scar Tissue}

The professional nurses massaged the scar above the wound, the breast and axillary tissues to relieve the scar tissues and thus reduce lymphatic obstruction caused by scar contracture.

\subsubsection{Manual Lymph Drainage}

Manual lymph drainage (MLD) is a massage therapy that requires gentle movements over the skin in a particular direction based on the structural characteristics of lymphatic system [7]. The massage was done in the manner of Stationary Circles, Rotary Technique or Scoop Technique from distal end to proximal end over the superficial lymph vessels. With midsternal line and spine as divide, massage was started from the wound to drain the lymph above the wound to axillary or supraclavicular lymph nodes on the other side; lymph below the wound to inguinal lymph nodes on the same side; lymph from medial upper arm of the anterior body through lateral upper arm to supraclavicular lymph nodes and lymph from medial upper arm of posterior body through lateral upper arm to dorsal lymph nodes or through dorsal to inguinal lymph nodes on the same side; lymph from the back of the hand, palm and forearm chelidon to lateral upper arm. The massage should be gentle enough to avoid local rubefaction. The principles are: the skin is touched by hand and pressure is light and alternating; massage is done in the desired direction of lymph movement and from proximal part to distal end; the movement is slow and rhythmic.

\subsubsection{Bandaging}

What followed manual lymph drainage was elastic bandaging for patients. The necessary tools including lotion, tubular bandage, polyester cotton pad and low-elastic bandage were prepared before bandaging. Detailed steps include: 1) the patients assumed a sitting or recumbent position and nurses used skin-friendly lotion to massage the affected limb carefully till the limb was moisturized enough to avoid damage. A piece of tubular pad, the length of which is twice the length stretching from hand back to shoulder was cut off and the affected limb was put into tubular pad without folding. 2) Bandaging for fingers: With the wrist as the anchor, the bandage was repeatedly wrapped around the finger to fix it. 3) Cotton pad bandaging for upper limb: Cut a small hole of $2-3 \mathrm{~cm}$ in diameter in the distant end of the cotton pad for the thumb to come out through it. A piece of soft cotton pad of $10 \mathrm{~cm}$ was used to wrap up the affected limb with an overlapping rate of $50 \%$. Folded foam pad was used to protect the elbow and ensure even pressure and then soft cotton pad of $15 \mathrm{~cm}$ in width was adopted to wrap the arm from below the elbow up towards the proximal end of the upper limb till the shoulder with edema. 4) Compression bandaging for upper arm and forearm: Pressure bandage of $6 \mathrm{~cm}$ in width was used for compression bandaging. The top of the bandage was fixed by wrapping up the wrist for a round, then the bandage moved from the back of the hand to the palm, and then moved back to the back of the hand. The bandage passed from lateral side of the thumb. To prevent a gap between the thumb and index finger, the edge of the upper bandage was pressed. Repeat the above steps, and 8-shape compression bandaging was performed on the palm with pressure bandage of $8 \mathrm{~cm}$ in width. 8 -shape compression 
bandaging was done from the wrist to the part below the elbow. 8-shape compression bandaging in opposite direction was performed from below the elbow to the wrist with bandage of $10 \mathrm{~cm}$ in width. The end of the bandage was fixed with tape and the end of the tubular bandage inside was flipped over the pressure bandage. Usually there were 5 layers of bandage wrapping up the affected forearm and 3 layers wrapping up the upper arm in the case of compression bandaging. The low-elastic bandage is required to accommodate 1 finger.

\subsubsection{Health Coaching}

After bandaging, professional nurses gave health coaching to patients mainly around the 18 guidelines for preventing lymphedema put forward by National Lymphedema Network (HLN) in 2003. The professional nurses mainly introduced to the patients and their family the detailed precautions for preventing lymphedema and self-care for the affected limb at home. Nurses also gave instructions on functional exercise, which could promote local blood circulation and lymphatic return, reducing lymphedema of the affected upper limb [9]. The patients could take functional exercise in spare time or in the intervals of work but should use pressure bandage or wear compression arm sleeves [10]. If no protection measures were taken when doing functional exercise, the edema might be aggravated.

\subsubsection{Functional Exercise}

1) Warm-up: activate large joints for 20-30 times; 2) Activate shoulder and improve muscle movement to promote lymphatic return to jugular vein. 3) Bend or stretch the affected upper limb and the lower limb on the other side at the same time. 4) Stretch exercise: raise the upper limb to touch the head to stretch the chest muscle and trapezius. 5) Breathing exercise: expand chest when breathing and put the palm of the healthy upper limb on the sternum to feel chest movement.6) Sing as a way of doing breathing exercise.

\subsection{Evaluation Methods}

\subsubsection{Measurement of Circumference}

Circumference measurement was done at transverse palmar crease, transverse carpal crease, $10 \mathrm{~cm}$ below transverse cubital crease, $10 \mathrm{~cm}$ above transverse cubital crease and $20 \mathrm{~cm}$ above transverse cubital crease before treatment and at the 7 th day, 14th day, 21st day after treatment.

\subsubsection{Life Quality of the Patients}

The Chinese version of FACT-B scale was used to evaluate the life quality of patients. The scale includes 36 items categorized into 5 aspects namely social/family status, physical condition, functional status, emotional status and additional attention [11]. The life quality was evaluated before treatment and after a course of treatment and was compared before and after treatment.

\subsection{Statistical Methods}

The software SPSS13.0 was adopted and the statistical data were expressed as $\bar{\chi}^{ \pm} \mathrm{s}$. Randomly designed variance analysis was employed for comparison of all the indicators before and after treatment. $\mathrm{P}<0.05$ indicates there is a significant difference.

\section{Results}

\subsection{Comparison of the Effect of CDT Treatment on the 28 Patients with Lymphedema in Affected Limb}

The circumference at transverse palmar crease, transverse carpal crease, $10 \mathrm{~cm}$ below transverse cubital crease, $10 \mathrm{~cm}$ above transverse cubital crease and $20 \mathrm{~cm}$ above transverse cubital crease after treatment were significantly lower than that before treatment. The detumescence of the middle segment of forearm (10 cm below transverse cubital crease) was better than that of the middle segment of upper arm (10 $\mathrm{cm}$ above transverse cubital crease) and of the superior segment of upper arm (20 cm above transverse cubital crease) with a significant difference $(\mathrm{P}<0.05)$ as shown in Table 1 .

Table 1. Comparison of the effect of CDT treatment on the 28 patients with lymphedema in affected limb.

\begin{tabular}{|c|c|c|c|c|c|c|}
\hline Time & Case & $\begin{array}{l}\text { Transverse palmar } \\
\text { crease }(\bar{\chi} \pm s)\end{array}$ & $\begin{array}{l}\text { Transverse carpal } \\
\text { crease }(\bar{\chi} \pm s)\end{array}$ & $\begin{array}{l}10 \mathrm{~cm} \text { below transverse } \\
\text { cubital crease }(\bar{\chi} \pm s)\end{array}$ & $\begin{array}{l}10 \mathrm{~cm} \text { above transverse } \\
\text { cubital crease }(\bar{\chi} \pm s)\end{array}$ & $\begin{array}{l}20 \mathrm{~cm} \text { above transverse } \\
\text { cubital crease }(\bar{\chi} \pm s)\end{array}$ \\
\hline $\begin{array}{l}\text { Before } \\
\text { treatment }\end{array}$ & 28 & $20 \pm 1.1$ & $21 \pm 1.9$ & $28 \pm 2.8$ & $31 \pm 4.3$ & $33 \pm 3.4$ \\
\hline $\begin{array}{l}1 \text { week after } \\
\text { treatment }\end{array}$ & 28 & $19.1 \pm 1.1$ & $19.1 \pm 1.5$ & $25.6 \pm 2.5^{*}$ & $29.2 \pm 3.7$ & $32.1 \pm 3.4$ \\
\hline $\begin{array}{l}2 \text { weeks after } \\
\text { treatment }\end{array}$ & 28 & $18.9 \pm 1.0$ & $18.4 \pm 1.7$ & $25.0 \pm 2.3^{*}$ & $28.7 \pm 3.8^{*}$ & $31.7 \pm 3.5^{*}$ \\
\hline
\end{tabular}

Notes: *: in comparison of that before treatment, $\mathrm{P}<0.05$; \#: in comparison of that 7 days after treatment, $\mathrm{P}<0.05$; $\diamond$ : in comparison of that 14 days after treatment, $\mathrm{P}<0.05$.

\subsection{Comparison of Life Quality Before and After CDT Treatment}

After 3 weeks of CDT treatment, the life quality of patients improved greatly and the difference in total score of FACT-B scale before and after treatment was statistically significant $(\mathrm{P}<0.05)$ as shown in Table 2. 
Table 2. Comparison of life quality before and after CDT treatment.

\begin{tabular}{llll}
\hline $\mathbf{n}$ & Time & FACT-B scale & $\boldsymbol{P}$ \\
\hline 28 & Before treatment & $108.22 \pm 9.29$ & \multirow{2}{*}{0.0056} \\
28 & 21 days after treatment & $112.96 \pm 8.21$ & \\
\hline
\end{tabular}

\section{Discussion}

Breast cancer is the most common female tumors. With the progress of medical technology, the cure rate and survival period of breast cancer is increasing continuously. Lymphedema as one of the most common complications of modified radical mastectomy can severely decrease the life quality of patients. CDT treatment is an effective therapy applied to lymphedema. It can effectively reduce edema and other lymphedema-induced symptoms [12].

In the current study we adopted manual lymph drainage as a decongestive therapy and found that after 3 weeks of CDT treatment, life quality of patients achieved great improvement and that the detumescence of the middle segment of forearm (10 cm below transverse cubital crease) was better than that of the middle segment of upper arm (10 $\mathrm{cm}$ above transverse cubital crease) and of the superior segment of upper arm $(20 \mathrm{~cm}$ above transverse cubital crease) with a significant difference, which was consistent with other related studies [8, 9, 13]. Some researchers performed manual lymph drainage on 6 patients with simple breast lymphedema and after 20 times of treatment the transverse diameter, vertical diameter and average diameter of breast steadily decreased at different times, and axillary web syndrome was cured. Thus, manual lymph drainage was thought as an effective decongestive therapy to improve the symptoms and signs of breast lymphedema after breast-conserving therapy $[14,15]$.

Complex decongestive therapy can improve quality of life for patients. It can open up lymph nodes, modify lymphatic return pathways and promote lymph circulation to reduce edema of the patients through massage [16]. Manual lymph drainage and low-elastic bandage are key elements of the treatment. Skin care and instruction on functional exercise are also necessary steps to achieve better therapeutic effects. We believe manual lymph drainage through massage over subaxillary lymph nodes can change lymphatic return pathways and prevent lymph from returning through congested lymph vessels so as to reduce edema of the patients. We also have adopted low-elastic bandage for compression bandaging which can produce stable and lasting pressure and thus effectively enhance the functions of lymph vessels and veins and lymph and blood circulation. Besides, to keep the skin clean and stick to functional exercise before and after treatment, avoid infection and promote lymphatic return are key to reducing and controlling lymphedema. Lymphedema treatment takes long time so in order to avoid relapse and maintain the therapeutic effect it is important to provide health coaching, spread preventive knowledge among patients and for patients to adhere to self-treatment.

\section{Conclusions}

In conclusion, CDT treatment for patients with lymphedema after radical mastectomy can significantly reduce lymphedema and improve quality of life for patients. It can open up lymph nodes, modify lymphatic return pathways and promote lymph circulation to reduce edema of the patients through massage. A combination of manual lymph drainage and other nursing measures can effectively prevent lymphedema, promote recovery of patients and thus is of clinical application value.

\section{Acknowledgements}

This work was financially supported by the Guangdong Provincia Medical Science and Technology Research Fund Project (NO. A2016165).

\section{References}

[1] Miller, K. D., Siegel, R. L., Lin, C. C., Mariotto, A. B., Kramer, J. L., \& Rowland, J. H., et al. (2016). Cancer treatment and survivorship statistics, 2016. CA: A Cancer Journal for Clinicians, 66 (4), 271-289.

[2] Hua, Alexandra, Sesto, Mary E., Zhang, Xiao, Wassenaar, Timothy R., \& Tevaarwerk, Amye J. (2019). Impact of survivorship care plans and planning on breast, colon, and prostate cancer survivors in a community oncology practice. Journal of Cancer Education (11), 1-7.

[3] Disipio, T., Rye, S., Newman, B., \& Hayes, S. (2013). Incidence of unilateral arm lymphoedema after breast cancer: a systematic review and meta-analysis. The Lancet Oncology, 14 (6): 500-515.

[4] Hyun, L. S., Yu-Sun, M., Yong, P. H., \& Tae-Du, J. (2012). Health-related quality of life in breast cancer patients with lymphedema who survived more than one year after surgery. Journal of Breast Cancer, 15 (4), 449-453.

[5] Pusic, A. L., Cemal, Y., \& Claudia Albornoz.... (2013). Quality of life among breast cancer patients with lymphedema: a systematic review of patient-reported outcome instruments and outcomes. Journal of Cancer Survivorship, 7 (1), 83-92.

[6] Hwang, J. M., Hwang, J. H., Kim, T. W., Chang, H. J., \& Chu, I. H. (2013). Long term effects of complex decongestive therapy in breast cancer patients with arm lymphedema after axillary dissection. PM\&R, 5 (9), S222.

[7] Pan YQ, Yang KH, Wang YL, et al. Massage interventions and treatment-related side effects of breast cancer: a systematic review and meta- analysis [J]. International Journal of Clinical Oncology, 2014, 19 (5): 829-841.

[8] Kang, Y., Jang, D. H., Jeon, J. Y., Lee, S. J., Jeong, S. Y., \& Shin, D. I., et al. (2012). Pressure monitoring of multilayer inelastic bandaging and the effect of padding in breast cancerrelated lymphedema patients. American Journal of Physical Medicine \& Rehabilitation, 91 (9), 768-773.

[9] Damstra, R. J., \& Partsch, H. (2009). Compression therapy in breast cancer-related lymphedema: a randomized, controlled comparative study of relation between volume and interface pressure changes. Journal of Vascular Surgery, 49 (5), 1256-1263. 
[10] Melgaard, \& Dorte. (2016). What is the effect of treating secondary lymphedema after breast cancer with complete decongestive physiotherapy when the bandage is replaced with kinesio textape?-a pilot study. Physiotherapy Theory and Practice, 1-6.

[11] Ng, R., Lee, C. F., Wong, N. S., Luo, N., Yap, Y. S., \& Lo, S. K., et al. (2012). Measurement properties of the english and chinese versions of the functional assessment of cancer therapy - breast (fact-b) in asian breast cancer patients. BREAST CANCER RESEARCH AND TREATMENT, 131 (2), 619-625.

[12] Stéphane Vignes, Porcher, R., Champagne, A., \& Dupuy, A. (2006). Predictive factors of response to intensive decongestive physiotherapy in upper limb lymphedema after breast cancer treatment: a cohort study. Breast Cancer Research \& Treatment, $98(1), 1-6$.

[13] Huang, T. W., Tseng, S. H., Lin, C. C., Bai, C. H., Chen, C. S., \& Hung, C. S., et al. (2013). Effects of manual lymphatic drainage on breast cancer-related lymphedema: a systematic review and meta-analysis of randomized controlled trials. World Journal of Surgical Oncology, 11 (1), 15-15.

[14] Zimmermann, A., Wozniewski, M., Szklarska, A., Lipowicz, A., \& Szuba, A. (2012). Efficacy of manual lymphatic drainage in preventing secondary lymphedema after breast cancer surgery. Lymphology, 45 (3), 103-12.

[15] Zhang, L., Fan, A., Yan, J., He, Y., Zhang, H., \& Zhang, H., et al. (2016). Combining manual lymph drainage with physical exercise after modified radical mastectomy effectively prevents upper limb lymphedema. Lymphatic Research and Biology, 14 (2): 104-8.

[16] Jain, P., Olszewski, W. L., Ambujam, G., Zaleska, M., \& Cakala, M.: (2009). Topography of accumulation of stagnant lymph and tissue fluid in soft tissues of human lymphedematous lower limbs. Lymphatic Research \& Biology, 7 (4), 239-245. 